\title{
Combining mercapto-functionalized palygorskite with zinc affect cadmium phytoavailability and soil microbial activity in rhizosphere soil
}

Yulong $\mathrm{Li}^{1}$, Chao Gao ${ }^{1}$, Sashuang Rong ${ }^{1}$, Jialin $\mathrm{Gu}^{2}$, Huiwei Zhao ${ }^{3}$, Shiming $\mathrm{Su}^{4}$, and wei $\mathrm{liu}^{1}$

\author{
${ }^{1}$ Hebei University \\ ${ }^{2}$ Beijing Academy of Agricultural and Forestry Sciences \\ ${ }^{3}$ National Semi-Arid Agricultural Technology Research Center \\ ${ }^{4}$ Chinese Academy of Agricultural Sciences
}

June 12, 2021

\begin{abstract}
Cadmium (Cd) pollution in soil pose a grave threat to human health. Combining various approaches to reduce Cd accumulation in crops is an active area of research to remediate farmlands with medium-high levels of Cd contamination. The Mercaptofunctionalized palygorskite (PGS-SH) and zinc $(\mathrm{Zn})$ application alone or in combination was investigated to explore reduction of Cd uptake in B. chinensis L and transformation of Cd in soil. The sole application of Zn or PGS-SH increased the biomass of B. chinensis L. and decreased the concentration of $\mathrm{Cd}$ in plants, but more improvements were observed from the combined application of Zn and PGS-SH. Low concentration of exogenous Zn $(50 \mathrm{mg} / \mathrm{kg})$ significantly increased the soil respiration rate (SRR) and the soil dehydrogenase activity (sDHA), while promoted B. chinensis L. growth while inhibiting Cd uptake. However, excessive exogenous Zn ([?] $200 \mathrm{mg} / \mathrm{kg}$ ) significantly inhibited B. chinensis L. growth and soil microbial activity. The combined application of PGS-SH and Zn had the highest sDHA (145.59\%) and lowest transport factor (TF) (27.59\%) compared with the CK. The combination of PGS-SH and Zn fertilizer is a safe and effective means for remediating Cd-contaminated soil and restoring microbial activity.
\end{abstract}

Highlights:

Mercapto-functionalized palygorskite is an excellent Cd stabilizer in soil.

Exogenous Zn significantly reduced Cd uptake in Brassica chinensis L.

Low-level exogenous Zn treatment $(50 \mathrm{mg} / \mathrm{kg}$ ) significantly increased the SRR and sDHA.

The combination of PGS-SH and $\mathrm{Zn}$ is feasible for remediation of Cd-contaminated soil. 


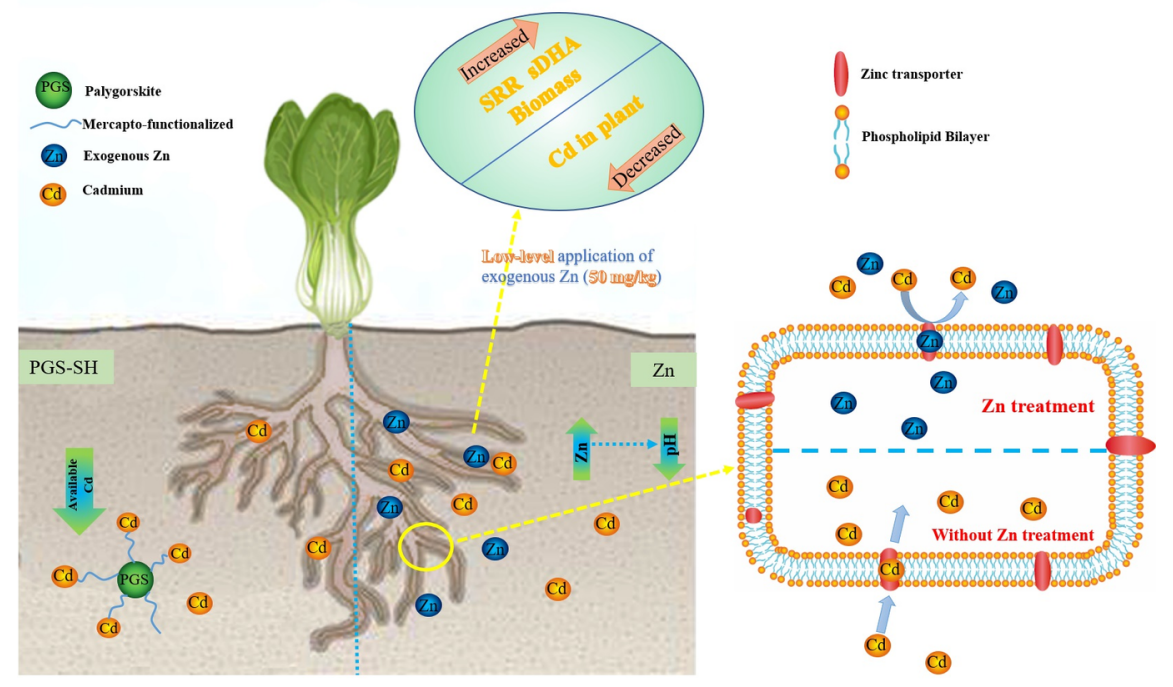

\section{Hosted file}

2021-3-16manuscript.docx available at https://authorea.com/users/419412/articles/ 525925-combining-mercapto-functionalized-palygorskite-with-zinc-affect-cadmiumphytoavailability-and-soil-microbial-activity-in-rhizosphere-soil

\section{Hosted file}

Tables and Figures.docx available at https://authorea.com/users/419412/articles/ 525925-combining-mercapto-functionalized-palygorskite-with-zinc-affect-cadmiumphytoavailability-and-soil-microbial-activity-in-rhizosphere-soil 